Artigo Original

Original Article

Adriana Ricarte ${ }^{1}$

Gisele Oliveira ${ }^{1}$

Mara Behlau'

Descritores

Voz

Qualidade de Vida

Estudos de validação

Tradução

Disfonia

Keywords

Voice

Quality of life

Validation studies

Translation

Dysphonia

Endereço para correspondência:

Adriana Ricarte

R. Hélio Pradines, 737/602, Ponta Verde,

Maceió (AL), Brasil, CEP: 57035-220.

E-mail: adriana_ricarte@hotmail.com

Recebido em: 20/07/2011

Aceito em: 13/03/2012

CoDAS 2013;25(3):242-9

\section{Validação do protocolo Perfil de Participação e Atividades Vocais no Brasil}

\author{
Validation of the Voice Activity and Participation Profile \\ protocol in Brazil
}

\section{RESUMO}

Objetivo: O objetivo deste estudo foi desenvolver a versão brasileira do protocolo Vocal Activity and Participation Profile (VAPP) e demonstrar suas propriedades de medida para que ele possa ser utilizado como um instrumento de autoavaliação da qualidade de vida de pacientes brasileiros com queixas vocais. Métodos: A tradução e validação foram realizadas seguindo as orientações do Scientific Advisory Committee of Medical Outcomes Trust. $\mathrm{O}$ instrumento foi aplicado em 50 pacientes, sendo 25 com queixa vocal e 25 sem queixa vocal. O instrumento foi submetido à adaptação linguística/cultural, validação, confiabilidade, reprodutibilidade e sensibilidade ao tratamento. O protocolo VAPP é composto por 28 questões distribuidas em cinco aspectos: autopercepção da intensidade do problema vocal, efeitos no trabalho, na comunicação diária, na comunicação social, e na emoção. Dois escores adicionais são inclusos: limitação das atividades (PLA) e restrição de participação (PRP). Resultados: Os resultados mostraram que a versão em português do protocolo VAPP, que recebeu o nome de PPAV - Perfil de Participação e Atividade Vocais é válida, confiável e apresenta um nível aceitável de reprodutibilidade, adotando-se $\mathrm{p}<0,05$. A resposta ao tratamento (sensibilidade) foi determinada por mudanças significativas entre os resultados pré e pós-tratamento (autopercepção da intensidade do problema vocal: 5,33/0,35; efeitos no trabalho: 12,40/1,93; efeitos na comunicação diária: 42,55/6,75; efeitos na comunicação social: 7,05/2,42; efeitos na emoção: 62,5/61,9; PLA: 33,3/2,81; PRP: 28,7/8,28). Conclusão: A versão brasileira do VAPP, o PPAV - Perfil de Participação e Atividade Vocais é válida, confiável e sensível à mudança, recebendo nome de Perfil de Participação e Atividades Vocais (PPAV). Portanto, o PPAV pode ser proposto como um instrumento útil para avaliar a qualidade de vida de pacientes disfônicos e resultados do tratamento.

\begin{abstract}
Purpose: The purpose of this study was to develop the Brazilian version of the Voice Activity and Participation Profile (VAPP) protocol and to demonstrate its measure properties so that it could be used as a self-evaluation instrument about the quality of life of Brazilian patients with vocal complaints. Methods: The translation and validation were performed following the guidelines of the Scientific Advisory Committee of Medical Outcomes Trust. The instrument was administered to 50 patients, 25 with vocal complaint and 25 without it. The instrument was submitted to linguistic/cultural adaptation, validation, reliability, reproducibility, and responsiveness. The VAPP protocol is composed of 28 questions distributed into five aspects: self-perception of the vocal problem intensity, effects at work, effects at daily communication, effects at social communication, and effects at emotion. Two additional scores are included: limitation in activities (LA) and participation restriction (PR). Results: The results showed that the Portuguese version of the VAPP protocol, called Perfil de Participação e Atividades Vocais (PPAV), is valid and reliable, presenting an acceptable reproducibility level, considering $\mathrm{p}<0.05$. Responsiveness to treatment was determined by significant changes between results before and after treatment (self-awareness of the vocal problem intensity: 5.33/0.35, effects at work: $12.40 / 1.93$, effects at daily communication: $42.55 / 6.75$, effects at social communication: 7.05/2.42, effects at emotion: 62.5/61.9; LA: 33.3/2.81; PR: 28.7/8.28). Conclusion: The Brazilian version of the VAPP protocol is valid, reliable, and responsive to changes. Therefore, it can be proposed as a useful instrument to evaluate quality of life of dysphonic patients and the treatment outcomes.
\end{abstract}

Trabalho realizado como conclusão do curso de especialização em voz, Centro de Estudos da Voz - CEV - São Paulo (SP), Brasil.

(1) Centro de Estudos da Voz - CEV - São Paulo (SP), Brasil.

Conflito de interesse: nada a declarar. 


\section{INTRODUÇÃO}

Segundo a Organização Mundial de Saúde (OMS), a saúde é um conceito multidimensional, que abrange não somente a ausência de doença, mas também os aspectos físicos, mentais e sociais $^{(1)}$. Esse conceito foi ampliado incorporando a concepção de qualidade de vida, que foi definida como a percepção que o indivíduo tem de sua posição na vida, levando em conta objetivos, expectativas, interesses pessoais e padrões sociais, culturais e econômicos ${ }^{(1)}$. $\mathrm{O}$ conceito de qualidade de vida foi introduzido há aproximadamente duas décadas como uma questão importante na prática clínica. Instrumentos que avaliam a qualidade de vida fornecem informação rápida sobre a percepção do indivíduo e sobre resultados do tratamento ${ }^{(2)}$.

As avaliações na área da saúde geralmente são feitas por meio de exames clínicos e/ou laboratoriais, embora a OMS recomende a inclusão do levantamento da perspectiva do indivíduo sobre seu estado de saúde na avaliação clínica ${ }^{(1)}$. Portanto, instrumentos que avaliam resultados de tratamento e a qualidade de vida devem ser cuidadosamente desenvolvidos e apresentar comprovadas medidas psicométricas de confiabilidade, validade e sensibilidade ${ }^{(3,4)}$. A maior parte dos instrumentos de qualidade de vida é desenvolvida em inglês e para que sejam validados em outro idioma, devem ser submetidos a uma adaptação cultural e linguística, com tradução cuidadosa seguindo-se normas pré-estabelecidas pela literatura e, em seguida, devem ter suas propriedades de medidas demonstradas em um contexto cultural específico ${ }^{(5)}$.

A qualidade de vida de um indivíduo pode ser afetada por condições que interfiram no seu bem-estar, sejam elas físicas ou psicológicas. Como a disfonia representa toda e qualquer dificuldade ou alteração na emissão vocal que impeça a produção natural da voz, ela pode afetar a qualidade de vida do indivíduo no que diz respeito aos aspectos pessoais, sociais e profissionais. De maneira ideal, o exame do paciente disfônico deve incluir uma anamnese detalhada, a avaliação otorrinolaringológica e a avaliação vocal ${ }^{(5)}$. A realização de tais avaliações não garantem a quantificação do problema de voz do paciente, de acordo com sua percepção ${ }^{(6)}$. Por vezes, as avaliações objetivas de uma disfonia podem apresentar resultados dentro dos limites da normalidade, enquanto protocolos de qualidade de vida e análises subjetivas oferecem diversas informações sobre o impacto da dificuldade na produção vocal. Recentemente, pesquisas mostraram a importância da inclusão de parâmetros subjetivos na avaliação vocal, não somente focados na percepção do avaliador profissional, mas também na autopercepção do paciente quanto ao impacto da alteração vocal na sua na vida ${ }^{(7,8)}$.

A escolha do protocolo Vocal Activity and Participation Profile - VAPP ${ }^{(9)}$ foi baseada a partir da importância de se utilizar no Brasil uma ferramenta de avaliação, que abordasse a diferença entre a limitação do problema vocal e a disposição do indivíduo em participar de atividades diárias, a fim de melhor conduzir o processo de tratamento. Embora haja outros protocolos no Brasil que avaliem o impacto de uma disfonia na qualidade de vida de um indivíduo com problema de voz, este é o único que contempla uma questão importante proposta pelo CIF - Classificação Internacional de Funcionalidade, Incapacidade e Saúde da Organização Mundial da Saúde, que é a participação e a limitação de atividades. Além disso, o VAPP é um dos únicos instrumentos que aborda aspectos no trabalho, na vida social, na vida diária e na expressão das emoções, oferecendo dessa maneira, mais detalhes que os demais instrumentos validados. Este protocolo tem sido usado em estudos que investigam a autopercepção que os indivíduos têm da sua voz, principalmente com a população que faz uso profissional da $v z^{(10-12)}$, e já foi validado em outras línguas ${ }^{(13)}$.

O objetivo deste estudo foi realizar a versão brasileira do protocolo VAPP, de autoavaliação do impacto de uma disfonia, desenvolvido em inglês, demonstrando suas propriedades de medida para que ele possa ser usado como um instrumento de avaliação de qualidade de vida de pacientes brasileiros com queixas vocais.

\section{MÉTODOS}

Este estudo foi avaliado e aprovado pelo Comitê de Ética em Pesquisa do Centro de Estudos da Voz (CEV), sob o parecer 4313/05. Todos os participantes foram orientados sobre os objetivos do estudo e concordaram em participar, assinando o Termo de Consentimento Livre e Esclarecido. Cabe ressaltar que a coleta de dados foi realizada no Hospital Beneficência Portuguesa de São Paulo.

O procedimento dessa pesquisa foi realizado em três etapas: tradução, adaptação cultural e linguística; caracterização clínica e demográfica; e avaliação das propriedades psicométricas, que foram desenvolvidas de acordo com as normas propostas pelo Scientific Advisory Commitee of the Medical Outcomes Trust ${ }^{(14)}$. A tradução do questionário foi realizada por três fonoaudiólogas bilíngues e a retrotradução (para o inglês) foi feita por outras duas fonoaudiólogas também bilíngues que não haviam participado da etapa anterior. As tradutoras foram informadas sobre o objetivo e o procedimento da pesquisa. Uma comissão formada por três especialistas em voz revisou as etapas e realizaram mudanças por consenso para produção do protocolo final.

Para realização da equivalência cultural e linguística, dez pacientes com queixa vocal que buscaram atendimento na Clínica de Otorrinolaringologia do Hospital Beneficência de São Paulo foram selecionados aleatoriamente, seis homens e quatro mulheres, com média de idade de 34 anos. Vale ressaltar que esses indivíduos não participaram da amostra utilizada para determinar a validação do protocolo. A cada uma das questões foi acrescentada a opção "não aplicável" para identificação de questões não compreendidas ou não apropriadas para a população, as quais seriam consideradas inválidas para a cultura em questão. Não houve necessidade de modificação ou eliminação de nenhuma das questões.

A versão final do instrumento (Figura 1) foi aplicada a 50 indivíduos, sendo que 25 com queixa vocal (11 homens e 14 mulheres, com idades entre 21 e 65 anos e média de idade de 37 anos), e 25 sem queixa vocal, que apresentavam queixas dermatológicas (sete homens e 18 mulheres, com idades entre 19 e 62 anos e média de idade de 37 anos). O grupo com 
queixa vocal foi formado por 25 sujeitos que buscaram atendimento no Hospital Beneficência Portuguesa de São Paulo, no setor de Otorrinolaringologia. Todos passaram por avaliação otorrinolaringológica e fonoaudiológica. Nenhum deles havia sido submetido a qualquer tratamento prévio para a voz, medicamentoso, cirúrgico ou fonoaudiológico. O grupo sem queixa vocal foi formado por 25 sujeitos sem queixa vocal que buscaram atendimento no Hospital Beneficência Portuguesa de São Paulo, por apresentarem queixas dermatológicas (como acne e manchas). Os critérios de exclusão adotados foram: idade menor de 18 anos e maior de 65 anos; e presença de outros distúrbios da comunicação.

O protocolo Vocal Activity and Participation Profile (VAPP) recebeu o nome em português de Perfil de Participação e Atividades Vocais (PPAV) ${ }^{(15)}$. É um questionário com 28 itens desenvolvidos especificamente para avaliar a qualidade de vida e o resultado de tratamentos dos transtornos vocais em indivíduos disfônicos. Os itens são distribuídos em cinco aspectos, que produzem uma pontuação específica, são estes: autopercepção da intensidade do problema vocal, com uma questão e pontuação máxima de 10 pontos; efeitos no trabalho, com quatro questões e pontuação máxima de 40 pontos; efeitos na comunicação diária, com doze questões e pontuação máxima de 120 pontos; efeitos na comunicação social, com quatro questões e pontuação máxima de 40 pontos; efeitos na emoção, com sete questões e pontuação máxima de 70 pontos. O protocolo ainda apresenta dois escores adicionais, chamados Pontuação de Limitação das Atividades (PLA) e a Pontuação de Restrição de Participação (PRP), com valores máximos de 100 pontos cada. Para o cálculo da PLA deve-se somar a pontuação das dez questões pares que envolvem os aspectos "trabalho", "comunicação diária" e "comunicação social" (questões 2, 4, 6, 8, 10, 12, 14, 16, 18 e 20); para o cálculo da PRP deve-se somar a pontuação das dez questões ímpares dos mesmos aspectos citados anteriormente (questões 3, 5, 7, 9, 11, 13, 15, 17, 19 e 21). Quanto maior a pontuação, maior é a limitação nas atividades vocais (dificuldades impostas nas atividades) e maior é a restrição na participação (indivíduo diminui ou evita participar de atividades que envolvam o uso da voz). O escore máximo deste protocolo, somando-se os escores de todas as questões, é de 280 pontos.

A caracterização clínica e demográfica dos participantes foi realizada de acordo com a idade, gênero, profissão, tipo de disfonia e presença de queixa vocal. Os indivíduos foram distribuídos em cinco grupos de acordo com a profissão ${ }^{(16)}$, relacionando o uso da voz com a demanda e o impacto de uma eventual disfonia: nível I - elite vocal, são os profissionais cujas carreiras podem ser seriamente afetadas devido a uma alteração vocal, como cantores e atores; nível II - profissional da voz falada para o qual uma alteração vocal de grau moderado poderia causar impacto profissional, como ocorre com a maioria dos professores; nível III - usuário não profissional da voz que teria suas funções profissionais prejudicadas somente por uma disfonia de grau acentuado, como médicos e vendedores; e finalmente, nível IV - usuário não profissional, não vocal, que não sofre limitações mesmo em condições extremas de comprometimento vocal, como desenhistas, programadores de computação, etc. Foi necessária a introdução de um outro nível, denominado $\mathrm{V}$, para os participantes desempregados, aposentados, estudantes e donas de casa.

Os pacientes do grupo com queixa vocal foram distribuídos em três grupos a partir da disfonia que apresentavam: funcional, organofuncional e orgânica, de acordo com os critérios utilizados para a classificação das disfonias, baseados em outro estudo $^{(17)}$. A categoria de disfonia funcional inclui pacientes com um desvio que tem o comportamento vocal como a base do desenvolvimento do problema vocal, incluindo alterações comportamentais, pequenas alterações estruturais e casos psicogênicos. A categoria de disfonia organofuncional inclui as lesões benignas, que decorrem diretamente do comportamento vocal, ou que este comportamento teve um papel importante na causa desta alteração, como nos casos de nódulos e pólipos. Por fim, a categoria de disfonia orgânica, que inclui disfonias neurológicas, endócrinas, tumores benignos ou carcinoma de laringe, que são distúrbios causados por uma série de processos que não dependem do uso da voz ${ }^{(17)}$.

A propriedade psicométrica de confiabilidade, que representa o grau pelo qual o protocolo está livre de erro aleatório ${ }^{(14)}$, foi analisada pela consistência interna, por meio do teste de correlação de Alfa de Cronbach e pela reprodutibilidade teste-reteste. A validade, que é o grau pelo qual o protocolo avalia o que se propõe avaliar ${ }^{(14)}$, foi determinada por meio da comparação dos escores do PPAV de ambos os grupos com a autoavaliação vocal representada, sendo obtida de três maneiras: relacionadas ao conteúdo, ao construto e ao critério. As duas primeiras são comumente realizadas na área de saúde, a terceira dificilmente é testada devido à falta de medidas amplamente aceitas. A sensibilidade ao tratamento reflete a habilidade do protocolo de identificar alterações importantes no problema para o qual é direcionado ${ }^{(14)} \mathrm{e}$ há a necessidade de comparar os grupos pré e pós-tratamento. Em nosso estudo, a sensibilidade do protocolo, foi demonstrada contemplando o primeiro aspecto do questionário por meio do teste dos Postos Sinalizados de Wilcoxon. A confiabilidade e a reprodutibilidade foram determinadas pelo teste-reteste e pela análise de consistência interna. O período de reteste variou de dois a 14 dias ${ }^{(7,18)}$. A reprodutibilidade foi determinada pelo teste dos Postos Sinalizados de Wilcoxon e a confiabilidade pelo o teste de coeficiente de correlação de Alfa de Cronbach. A sensibilidade ao tratamento foi avaliada comparando-se os escores pré e pós-terapia fonoaudiológica, por meio do Teste dos Postos Sinalizados de Wilcoxon.

Para o tratamento estatístico, utilizou-se o programa SPSS Statistical Package for Social Sciences, versão 13.0. O nível de significância adotado para este estudo foi de $5 \%(\mathrm{p}<0,05)$.

\section{RESULTADOS}

As características demográficas foram semelhantes nos dois grupos. Os escores do protocolo PPAV foram apresentados de acordo com o tipo de disfonia e uso profissional da voz para o grupo com queixa vocal (Tabela 1). 
Tabela 1. Escores médios do grupo de indivíduos com queixa vocal de acordo com o tipo de disfonia e uso profissional da voz

\begin{tabular}{|c|c|c|c|c|c|c|c|c|}
\hline Variáveis & Aspecto 1 & Aspecto 2 & Aspecto 3 & Aspecto 4 & Aspecto 5 & Total $^{*}$ & PLA & PRP \\
\hline \multicolumn{9}{|l|}{ Disfonia } \\
\hline Funcional & 5,41 & 15,10 & 27,59 & 12,26 & 19,59 & 79,94 & 31,68 & 23,25 \\
\hline Orgânica & 6,13 & 6,50 & 47,20 & 6,17 & 7,23 & 73,23 & 25,93 & 33,93 \\
\hline Organofuncional & 5,41 & 13,91 & 49,47 & 14,18 & 29,76 & 112,73 & 43,31 & 34,24 \\
\hline \multicolumn{9}{|l|}{ Profissão } \\
\hline Nível I & 6,73 & 24,07 & 41,40 & 9,57 & 34,30 & 116,07 & 19,80 & 6,50 \\
\hline Nível II & 4,34 & 13,39 & 41,83 & 13,06 & 17,60 & 90,21 & 48,53 & 39,46 \\
\hline Nível III & 1,64 & 4,52 & 12,91 & 3,92 & 8,46 & 31,45 & 36,76 & 30,58 \\
\hline Nível IV & 3,04 & 2,06 & 9,18 & 3,26 & 4,76 & 22,30 & 23,98 & 13,68 \\
\hline Nível V & 2,93 & 4,88 & 28,26 & 8,07 & 14,23 & 58,36 & 50,68 & 45,82 \\
\hline
\end{tabular}

*Soma das médias dos aspectos

Legenda: Aspecto 1 = autopercepção do problema de voz; Aspecto 2 = efeitos no trabalho; Aspecto 3 = efeitos na comunicação diária; Aspecto 4 = efeitos na comunicação social; Aspecto 5 = efeitos na emoção; PLA = pontuação de limitação nas atividades; PRP = pontuação de restrição na participação

Tabela 2. Coeficiente de correlação e significância dos grupos de indivíduos com e sem queixa vocal de acordo com a autopercepção da intensidade do problema vocal para cálculo da validade do protocolo

\begin{tabular}{|c|c|c|c|c|c|c|}
\hline \multirow{3}{*}{ Aspectos PPAV } & \multicolumn{6}{|c|}{ Autopercepção da intensidade do seu problema vocal } \\
\hline & \multicolumn{3}{|c|}{ Com queixa vocal } & \multicolumn{3}{|c|}{ Sem queixa vocal } \\
\hline & Média & Coeficiente & Valor de $p$ & Média & Coeficiente & Valor de $p$ \\
\hline Efeito no trabalho & 13,3 & 0,446 & $0,026^{*}$ & 0,2 & 0,912 & $<0,001^{*}$ \\
\hline Efeito na comunicação diária & 43,1 & 0,546 & $0,005^{*}$ & 0,9 & 0,686 & $<0,001^{*}$ \\
\hline Efeito na comunicação social & 12,7 & 0,474 & $0,017^{*}$ & 0,2 & 0,864 & $<0,001^{*}$ \\
\hline Efeito na emoção & 24,2 & 0,677 & $<0,001^{*}$ & 0,4 & 0,777 & $<0,001^{*}$ \\
\hline Total & 98,8 & 0,693 & $<0,001^{*}$ & 1,7 & 0,759 & $<0,001^{*}$ \\
\hline PLA & 37,9 & 0,731 & $<0,001^{*}$ & 0,66 & 0,674 & $<0,001^{*}$ \\
\hline PRP & 31,1 & 0,440 & $0,028^{*}$ & 0,66 & 0,740 & $<0,001^{*}$ \\
\hline
\end{tabular}

*Valores significativos $(p<0,05)$ - Teste de Correlação de Spearman

Legenda: PLA = pontuação de limitação nas atividades; PRP = pontuação de restrição na participação

\section{Validade}

Os escores do protocolo PPAV foram comparados com a pontuação da autopercepção da intensidade do problema vocal, nos grupos de indivíduos com e sem queixa vocal. Assim, foi realizado o cálculo da validade do protocolo, por meio do teste de correlação de Spearman (Tabela 2). $\mathrm{O}$ instrumento mostrou diferença entre os dois grupos, pois os pacientes com queixa vocal apresentaram maiores escores do que os pacientes sem queixa.

\section{Confiabilidade}

A confiabilidade do instrumento foi demonstrada pela determinação da consistência interna (Tabela 3 ). $\mathrm{O}$ valor obtido pelo teste em cada aspecto foi elevado, tanto no escore total quanto nos escores adicionais. Os coeficientes de correlação de cada aspecto e escores parciais demonstraram correlação positiva. A comparação dos valores de teste-reteste apresentou um nível aceitável de reprodutibilidade (Tabela 4).

\section{Sensibilidade ao tratamento}

A capacidade de avaliar a resposta de um tratamento por meio do protocolo foi testada, comparando-se os resultados pré e pós-terapia fonoaudiológica (Tabela 5). Nas mudanças após o
Tabela 3. Cálculo de confiabilidade do Perfil de Participação e Atividades Vocais: consistência interna dos aspectos e escores totais e adicionais

\begin{tabular}{lcc}
\hline Aspectos & $\begin{array}{c}\text { Coeficiente } \\
\text { de Alfa de } \\
\text { Cronbach }\end{array}$ & Valor de p \\
\hline $\begin{array}{l}\text { Autopercepção da intensidade } \\
\text { do problema vocal }\end{array}$ & 0,812 & $<0,001^{*}$ \\
Efeitos no trabalho & 0,896 & $<0,001^{*}$ \\
Efeitos na comunicação diária & 0,920 & $<0,001^{*}$ \\
Efeitos na comunicação social & 0,803 & $<0,001^{*}$ \\
Efeitos na emoção & 0,908 & $<0,001^{*}$ \\
Total & 0,754 & $<0,001^{*}$ \\
PLA & 0,920 & $<0,001^{*}$ \\
PRP & 0,812 & $<0,001^{*}$ \\
\hline
\end{tabular}

tratamento houve diferença para o escore total, autopercepção da intensidade do problema vocal, efeitos na comunicação social, efeito nas emoções e pontuação de limitação de atividades.

\section{DISCUSSÃO}

O desenvolvimento e a validação de instrumentos que medem a qualidade de vida tornaram-se um importante foco em diferentes áreas no campo da saúde. Tais instrumentos devem ser submetidos à avaliação em diferentes situações, 
para que suas propriedades de medidas possam ser demons$\operatorname{tradas}^{(3,19,20)}$. A maioria dos instrumentos de avaliação da qualidade de vida foi desenvolvida em inglês ${ }^{(21)}$. Portanto, o instrumento deve ser traduzido de acordo com diretrizes pré-estabelecidas na literatura para o uso em outras línguas. Os instrumentos não devem ser apenas traduzidos, mas também apresentarem suas medidas psicométricas testadas em um contexto cultural específico ${ }^{(3,14)}$.

A avaliação da qualidade de vida é relevante não apenas para traçar linhas para atuação médica, mas também para demonstrar a efetividade e o desempenho dos avanços atuais na medicina, melhorando o exercício profissional na área de saúde ${ }^{(22)}$.

Os resultados deste estudo mostraram que os indivíduos com disfonias organofuncionais relataram maior impacto na sua qualidade de vida, apresentando escores do protocolo PPAV mais elevados, quando comparados aos indivíduos com outros tipos de disfonia. Talvez esse resultado se deva ao fato de que uma alteração organofuncional seja formada pela associação de um problema comportamental e uma lesão nas pregas vocais, o que pode limitar o desempenho vocal desses indivíduos. As disfonias funcionais e orgânicas

*Valores significativos $(p<0,05)$ - Coeficientes de Correlação alfa de Cronbach Legenda: PLA = pontuação de limitação nas atividades; PRP = pontuação de restrição na participação

Tabela 4. Cálculo de reprodutibilidade para o Perfil de Participação e Atividades Vocais: teste-reteste para reprodutibilidade por aspectos. Escore total e cálculos adicionais

\begin{tabular}{|c|c|c|c|}
\hline Aspectos & Média & $\begin{array}{l}\text { Desvio- } \\
\text { padrão }\end{array}$ & Valor de $p$ \\
\hline \multicolumn{4}{|c|}{ Autopercepção da intensidade } \\
\hline Teste & 5,50 & 2,51 & \multirow{2}{*}{0,164} \\
\hline Reteste & 5,56 & 2,36 & \\
\hline \multicolumn{4}{|l|}{ Trabalho } \\
\hline Teste & 13,36 & 11,81 & \multirow{2}{*}{0,246} \\
\hline Reteste & 13,48 & 11,64 & \\
\hline \multicolumn{4}{|c|}{ Comunicação diária } \\
\hline Teste & 43,07 & 29,67 & \multirow[t]{2}{*}{0345} \\
\hline Reteste & 43,38 & 29,25 & \\
\hline \multicolumn{4}{|c|}{ Comunicação social } \\
\hline Teste & 12,68 & 10,84 & \multirow[t]{2}{*}{0,618} \\
\hline Reteste & 12,56 & 11,11 & \\
\hline \multicolumn{4}{|l|}{ Emoção } \\
\hline Teste & 24,21 & 17,73 & \multirow[t]{2}{*}{0,367} \\
\hline Reteste & 23,06 & 18,13 & \\
\hline \multicolumn{4}{|l|}{ Total } \\
\hline Teste & 98,81 & 60,63 & \multirow[t]{2}{*}{0,545} \\
\hline Reteste & 98,04 & 59,08 & \\
\hline \multicolumn{4}{|l|}{ PLA } \\
\hline Teste & 37,97 & 23,17 & \multirow[t]{2}{*}{0,053} \\
\hline Reteste & 38,39 & 23,38 & \\
\hline \multicolumn{4}{|l|}{ PRP } \\
\hline Teste & 31,13 & 25,23 & \multirow{2}{*}{0,957} \\
\hline Reteste & 25,23 & 24,52 & \\
\hline
\end{tabular}

Teste dos Postos Sinalizados de Wilcoxon $(p<0,05)$

Legenda: PLA = pontuação de limitação nas atividades; PRP = pontuação de restrição na participação também apresentaram escores elevados, reforçando o impacto negativo de uma disfonia em diversos aspectos da vida do falante. As profissões de nível I (cantores e atores) apresentaram as maiores médias de escore total, não necessariamente porque estes profissionais tenham grandes desvios, mas sim porque a disfonia pode ser um agente limitante para o bom rendimento profissional. Outra hipótese para o escore mais elevado entre os demais grupos é que talvez estes indivíduos considerem importante qualquer sintoma da disfonia, e por isso, recorrem à ajuda especializada precocemente, para que o problema não venha a prejudicar sua vida profissional e/ou social $^{(23)}$ (Tabela 1$)$.

A versão brasileira do VAPP apresentou um bom desempenho como um instrumento de avaliação vocal em populações de pacientes disfônicos. A validade do instrumento foi demonstrada pela sólida relação entre a autopercepção da intensidade do problema vocal e os escores médios dos aspectos e cálculos adicionais do protocolo PPAV, bem como pelas diferenças significativas obtidas entre os grupos de indivíduos com e sem queixa vocal (Tabela 2). Além disso, os resultados mostraram uma correlação positiva entre a autopercepção vocal e os escores

Tabela 5. Cálculo para sensibilidade ao tratamento do Perfil de Participação e Atividades Vocais: diferença da média dos escores do protocolo Perfil de Participação e Atividades Vocais no grupo de indivíduos com queixa vocal, pré e pós-terapia

\begin{tabular}{|c|c|c|c|}
\hline Aspectos & Média & Desvio-padrão & Valor de $p$ \\
\hline \multicolumn{4}{|c|}{ Autopercepção da intensidade } \\
\hline Pré-terapia & 5,33 & 2,69 & \multirow{2}{*}{$0,028^{*}$} \\
\hline Pós-terapia & 0,35 & 0,15 & \\
\hline \multicolumn{4}{|l|}{ Trabalho } \\
\hline Pré-terapia & 12,40 & 12,49 & \multirow{2}{*}{0,116} \\
\hline Pós-terapia & 1,93 & 0,92 & \\
\hline \multicolumn{4}{|c|}{ Comunicação diária } \\
\hline Pré-terapia & 42,55 & 30,85 & \multirow{2}{*}{$0,046^{*}$} \\
\hline Pós-terapia & 6,75 & 2,21 & \\
\hline \multicolumn{4}{|c|}{ Comunicação social } \\
\hline Pré-terapia & 7,05 & 6,93 & \multirow{2}{*}{0,249} \\
\hline Pós-terapia & 2,42 & 1,12 & \\
\hline \multicolumn{4}{|l|}{ Emoção } \\
\hline Pré-terapia & 15,65 & 16,28 & \multirow{2}{*}{$0,046^{*}$} \\
\hline Pós-terapia & 1,57 & 0,82 & \\
\hline \multicolumn{4}{|l|}{ Total } \\
\hline Pré-terapia & 82,98 & 63,34 & \multirow{2}{*}{$0,046^{*}$} \\
\hline Pós-terapia & 13,02 & 4,55 & \\
\hline \multicolumn{4}{|l|}{ PLA } \\
\hline Pré-terapia & 33,3 & 28,45 & \multirow{2}{*}{$0,046^{*}$} \\
\hline Pós-terapia & 2,81 & 1,39 & \\
\hline \multicolumn{4}{|l|}{ PRP } \\
\hline Pré-terapia & 28,7 & 28,92 & \multirow{2}{*}{0,249} \\
\hline Pós-terapia & 8,28 & 3,23 & \\
\hline
\end{tabular}

*Valores significativos $(p<0,05)$ - Teste dos Postos Sinalizados de Wilcoxon Legenda: PLA = pontuação de limitação nas atividades; PRP = pontuação de restrição na participação 
Protocolo do perfil de participação e atividades vocais - PPAV

Autopercepção do grau de seu problema vocal

O quanto o seu problema de voz é intenso?

Normal I I Intenso

Efeitos no trabalho

Seu trabalho é afetado pelo seu problema de voz?

Nunca I

I Sempre

Nos últimos 6 meses você chegou a pensar em mudar seu trabalho por causa do seu problema de voz?

Nunca I I Sempre

Seu problema de voz criou alguma pressão em seu trabalho?

Nunca I

I Sempre

Nos últimos 6 meses, o seu problema de voz tem afetado o futuro de sua carreira profissional?

Nunca I Sempre

Efeitos na comunicação diária

As pessoas pedem para você repetir o que acabou de dizer por causa do seu problema de voz?

Nunca I

I Sempre

Nos últimos 6 meses você alguma vez evitou falar com as pessoas por causa do seu problema de voz?

Nunca I I Sempre

As pessoas têm dificuldade de compreender você ao telefone por causa do seu problema de voz?

Nunca I__ I Sempre

Nos últimos 6 meses você reduziu o uso do telefone por causa do seu problema de voz?

Nunca I___ I Sempre

O seu problema de voz afeta sua comunicação em ambientes silenciosos?

Nunca I___ Sempre

Nos últimos 6 meses você chegou a evitar conversas em ambientes silenciosos por causa do seu problema de voz?

Nunca I___ Sempre

O seu problema de voz afeta sua comunicação em ambientes ruidosos?

Nunca I__ I Sempre

Nos últimos 6 meses você alguma vez chegou a evitar conversas em ambientes ruidosos por causa do seu problema de voz?

Nunca I___ Sempre

Seu problema de voz afeta sua mensagem quando você está falando para um grupo de pessoas?

Nunca I__ I Sempre

Nos últimos 6 meses você alguma vez evitou conversas em grupo por causa do seu problema de voz?

Nunca I

I Sempre

O seu problema de voz afeta na transmissão da sua mensagem?

Nunca I

I Sempre

Nos últimos 6 meses você alguma vez evitou falar por causa do seu problema de voz?

Nunca I

I Sempre

Efeitos na comunicação social

Seu problema de voz afeta suas atividades sociais?

Nunca I

I Sempre

Nos últimos 6 meses você evitou atividades sociais por causa do seu problema de voz?

Nunca I

I Sempre

Sua família, amigos ou colegas de trabalho se incomodam com seu problema de voz?

Nunca I

I Sempre

Nos últimos 6 meses alguma vez você evitou comunicar-se com seus familiares, amigos ou colegas de trabalho por causa do seu problema de voz?

Nunca I I Sempre

Efeitos na sua emoção

Você se sente chateado por causa do seu problema de voz?

Nunca I

I Sempre

Você está envergonhado pelo seu problema de voz?

Nunca I

Você está com baixa autoestima por causa do seu problema de voz?

Nunca I Sempre

Você está

I Sempre

Nunca I I Sempre

Você se sente insatisfeito por causa da sua voz?

Nunca I Sempre

Seu problema de voz afeta sua personalidade?

Nunca I I Sempre

Seu problema de voz afeta sua autoimagem?

Nunca I

I Sempre

Figura 1. Versão brasileira do protocolo PPAV (Ricarte, Oliveira e Behlau, 2006) 
do protocolo PPAV, sendo que quanto pior a autopercepção vocal, maior é a pontuação no protocolo. Tal correlação também foi observada no estudo original ${ }^{(9)}$. Os dados do estudo indicam que o protocolo PPAV é um instrumento sensível para identificar distúrbios vocais. Na comparação entre os grupos, os escores obtidos pelos indivíduos sem queixa foram inferiores aos obtidos pelos indivíduos sem queixa. Resultados similares também foram observados em outros estudos ${ }^{(15,24,25)}$, nos quais indivíduos disfônicos apresentaram escores mais elevados que indivíduos do grupo controle.

A confiabilidade do protocolo foi demonstrada pela determinação da consistência interna (Tabela 3 ) e da reprodutibilidade teste-reteste (Tabela 4). A consistência interna do PPAV foi determinada por meios dos valores de Alfa de Cronbach estatisticamente elevados. Para reprodutibilidade o protocolo foi aplicado duas vezes sem que o paciente tivesse sido submetido a qualquer tratamento, uma vez para a obtenção dos valores do teste e outra para os valores de reteste. A comparação dos valores teste-reteste, por meio do Teste dos Postos Sinalizados de Wilcoxon, mostrou um nível aceitável de reprodutibilidade, pois os resultados nos dois momentos foram semelhantes (Tabela 4). Portanto, a confiabilidade do protocolo foi determinada por meio da demonstração de sua consistência interna e de sua reprodutibilidade, como observado também no estudo de validação do questionário original ${ }^{(9)}$.

As mudanças na voz após o tratamento são apresentadas pelos escores médios do protocolo PPAV (Tabela 5). O presente estudo demonstrou que o protocolo PPAV é um instrumento sensível ao tratamento. Houve diferenças na maioria dos aspectos do PPAV quando foram comparados os escores pré e pós-tratamento fonoaudiológico. Provavelmente todos os escores não tenham apresentado mudanças expressivas devido ao número de indivíduos submetidos ao tratamento, além do fato de que a décima sessão de fonoterapia não necessariamente correspondeu à alta fonoaudiológica. Contudo, os resultados foram suficientes para demonstrar a sensibilidade ao tratamento, uma vez que as mudanças observadas são indicativas de melhora do padrão vocal sob a percepção do paciente e de uma redução nos efeitos negativos de um problema de voz na qualidade de vida.

O protocolo ainda apresenta dois cálculos adicionais, denominados de: PLA e PRP. Os resultados desses cálculos geram respostas fundamentais para o bom desempenho na terapia, pois a partir deles o clínico consegue saber se o paciente se priva de executar suas atividades ou modifica o seu desempenho. Dessa forma, é possível a obtenção de dados que podem ser diretamente abordados em terapia. Nesta pesquisa, os participantes que apresentaram queixa vocal demonstraram maior limitação em exercer as atividades profissionais, assim como as demais atividades do dia a dia, em decorrência do problema vocal, do que diretamente restritos a executá-las. Outros autores que utilizaram o PPAV também observaram este padrão de resposta ${ }^{(10,26)}$.

A importância do desenvolvimento e validação de instrumentos que avaliam o efeito de uma alteração vocal na qualidade de vida de um indivíduo é a principal etapa de um processo extenso e indispensável para estabelecer medidas de autopercepção. Tais instrumentos constituem uma parte fundamental na avaliação do paciente com queixa vocal, pois fornecem dados referentes à evolução do tratamento.

\section{CONCLUSÃO}

A versão brasileira do protocolo VAPP é válida, confiável e sensível. É um instrumento voltado especificamente a indivíduos com alterações vocais e, desta forma, mostra-se útil para avaliar a qualidade de vida dos pacientes disfônicos e os resultados dos tratamentos.

*AR foi responsável por coletar e tabular datos, analisar e redigir o manuscrito; $G O$ ficou encarregado de analisar e corrigir o manuscrito; $M B$ foi o orientador e responsável pela revisão científica e final.

\section{REFERÊNCIAS}

1. World Health Organization. (1997). World Health Organization Quality: Measuring Quality of Life. Geneva: World Health Organization. WHO/ MSA/MNH/PSF/97.4.

2. Ciconelli RM, Ferraz MB, Santos W, Meinão I, Quaresma MR. Tradução para a língua portuguesa e validação do questionário genérico de avaliação da qualidade de vida SF-36 (Brasil SF-36). Rev Bras Reumatol. 1999;39(3):143-50.

3. Guillemin F, Bombardier C, Beaton D. Cross-cultural adaptation of health-related quality of life measures: literature review and proposed guidelines. J Clin Epidemiol. 1993;46(12):1417-32.

4. Gill TM, Feinstein AR. A critical appraisal of the quality of quality-of-life measurements. JAMA. 1994Aug; 272(8):619-26.

5. Gasparini G, Behlau M. Quality of life: validation of the Brazilian version of the voice-related quality of life (V-RQOL) measure. J Voice. 2009Jan;23(1):76-81

6. Benninger MS, Ahuja AS, Gardner G, Grywalski C. Assessing outcomes for dysphonic patients. J Voice. 1998;12(4):540-50.

7. Hogikyan ND, Sethuraman G. Validation of an instrument to measure voice-related quality of life - V-RQOL . J Voice. 1999Dec;13(4):557-69.

8. Hogikyan ND. The voice-related quality of life (V-RQOL) measure: history and ongoing utility of a validated voice outcomes instrument. Voice and Voice Disorders. 2004;14(1):3-5.

9. Ma EP, Yiu EM. Voice activity and participation profile: assessing the impact of voice disorders on daily activities. J Speech Lang Hear Res. 2001;44(3):511-24.

10. Ricarte A, Bommarito S, Chiari B. Impacto vocal de professores. Rev CEFAC. 2011;13(4):719-27.

11. Ma EP, Yiu EM. Scaling voice activity limitation and participation restriction in dysphonic individuals. Folia Phoniatr Logop. 2007; 59(2):74-82.

12. Konnai RM, Jayaram M, Scherer RC. Developmente and validation of a voice disorder outcome profile for an Indian population. J Voice. 2010Mar;24(2):206-20

13. Sukanen O, Sihvo M, Rorarius E, Lehtihalmes M, Autio V, Kleemola L. Voice Activity and Participation Profile (VAPP) in assessing the effects of voice disorderes on pacients' quality of life: validity and reliability of the Finnish version of VAPP. Logoped Phoniatr Vocol. 2007; 32(1):3-8

14. Scientific Advisory Committee of Medical Outcomes Trust. Assessing health status and quality-of-life instruments: attributes and review criteria. Qual Life Res. 2002:11(3):193-205.

15.Behlau M, Santos LMA, Oliveira G. Cross-cultural adaptation and validation of the voice handicap index into Brazilian Portuguese. J Voice. 2011;25(3):354-9. 
16. Koufman JA, Isaacson G. Voice disorders. Otolaryngol Clin North Am. 1991;24(5):989-98.

17. Behlau B, Madazio G, Feijó D, Pontes PAL. Avaliação da voz. In: Behlau, B. Voz: o livro do especialista. São Paulo: Revinter, 2001. p. 83-245.

18. Helidoni ME, Murry T, Moschandreas J, Lionis C, Printza A, Velegrakis GA. Cross-cultural adaptation and validation of the voice handicap index into Greek. J Voice. 2010;24(2):221-7.

19. Guyatt GH, Veldhuyzen Van Zanten SJ, Feeny DH, Patrick DL. Measuring quality of life in clinical trials: a taxonomy and review. CMAJ. 1989;140(12):1441-8.

20. Barbotte E, Guillemin F, Chau N, Lorhandicap Group. Prevalence of impairments, disabilities, handicaps and quality of life in the general population: a review of recent literature. Bull World Health Organ. 2001;79(11):1047-55.
21. Lam PK, Chan KM, Ho WK, Kwong E, Yiu EM, Wei WI. Cross-cultural adaptation and validation of the Chinese Voice Handicap Index-10. Laryngoscope. 2006;116(7):1192-8.

22. Roper WL, Winkenwerder W, Hackbarth GM, Krakauer H. Effectiveness in health care: an initiative to evaluate and improve medical practice. $\mathrm{N}$ Engl J Med. 1988;319(18):1197-202.

23. Putnoki DS, Hara F, Oliveira G, Behlau M. Qualidade de vida em voz: o impacto de uma disfonia de acordo com o gênero, idade e uso vocal profissional. Rev Soc Bras Fonoaudiol. 2010;15(4):485-90.

24. Amir O, Ashkenazi O, Leibovitzh T, Michael O, Tavor Y, Wolf M. Applying the Voice Handicap Index (VHI) to dysphonic and nondysphonic Hebrew speakers. J Voice. 2006;20(2):318-24.

25. Guimarães I, Abberton E. An investigation of the Voice Handicap Index with speakers of Portuguese: preliminary data. J Voice. 2004;18(1):71-82.

26. Kasama ST, Brassolotto AG. Percepção vocal e qualidade de vida. Pro Fono. 2007;19(1):19-28. 